\title{
A Study of Organisms Causing Surgical Site Infections and their Antimicrobial Susceptibility in a Tertiary Care Hospital
}

\author{
R. Aruthra and P. Neelusree* \\ Saveetha Medical College, Thandalam, India \\ *Corresponding author
}

\section{A B S T R A C T}

\section{Ke y w o r ds \\ Antimicrobial susceptibility, Surgical site infection, Antibiotic resistance \\ Article Info \\ Accepted: \\ 04 August 2019 \\ Available Online: \\ 10 September 2019}

\begin{abstract}
Surgical antimicrobial prophylaxis refers to a brief case of antibiotic given during post surgery. Surgical site infections increase the hospital stay and prophylaxis has the potential to shorten the hospital stay. Surgical site infection is one of the most common post operative complication and causes significant post- operative morbidity and mortality. The objective of our study was to study the various microorganisms causing surgical site infection, to study their antibiotic susceptibility pattern. This was a postoperative retrospective series study, with 130 patients irrespective of age or gender, presented to the surgical ward of Saveetha Medical College (tertiary care hospital) and undergoing surgical intervention, conducted from Nov 2018 to Feb 2019. Out of 130 patients analysed the most prevalent microorganism is E. coli. The resistance of various microorganisms is studied. Carbapenems would be a better drug for gram negative coverage and vancomycin and linizolid would be a better drug for gram positive coverage.
\end{abstract}

\section{Introduction}

Surgical site infection is the infections of the tissues, organs or spaces exposed by surgeons during performance of and invasive procedure. SSI are characterized by a breach of mechanical / anatomical defence mechanism (barriers) and are associated with greater morbidity, mortality, increases cost of care. SSI can increase the length of time a patient stays in a hospital and thereby increase the cost of health care, not only the patient but his/her family also suffers.
This study was done to study the common micro organisms causing surgical site infections and to study the antimicrobial susceptibility pattern of those organisms.

\section{Materials and Methods}

Retrospective study carried out on 130 post operative surgical site patients. This study involved Department of surgery, Dept of Microbiology, Saveetha Medical Hospital, Thandalam. This study involved patients with SSI within the period of November 2018February 2019. 
Various samples received from these patients (pus, wound swab, drain, discharge from various post operative sites) sent to Microbiology lab for gram stain, Acid fast stain, culture and sensitivity were included in this study.

All these samples were processed in Microbiology lab through standard methods were analysed in our study for the common etiological agents and the antibiotic susceptibility pattern.

\section{Results and Discussion}

Retrospective study carried out by the Department of surgery and department of Microbiology on surgical site infections to analyse the common organisms that can be isolated from surgical site and to analyse the common antibiotics that would be effective against those organisms.

Various specimens were collected from 130 patients from the surgical wards of Saveetha medical hospital, Thandalam are listed below (Table 1, Figure 1) shows the details of various specimens collected.

The common microorganisms causing SSI in our tertiary care hospital are listed below
(Table 2, Figure 2). These microorganisms are the common organisms causing surgical site infections as per other studies done in different areas of interest.

There are wide range of antibiotics used for treatment of gram positive and gram negative organisms. The unwanted usage of antibiotics has resulted in increased resistance among the common microorganisms causing SSI which makes treatment of post operative patients difficult. The resistance patterns of common microorganisms causing SSI in our setting are listed below (Table 3).

The antimicrobial susceptibility pattern of most common microorganisms both gram positive and gram negative organisms causing SSI in our hospital are listed (Table 4). This study mainly focuses on the common gram positive and gram negative organism and the antibiotic coverage of those gram positive and gram negative organism.

In the present study showed carbapenems had a better coverage against all the common gram negative organisms. Vancomycin and linizolid had a better coverage against the common gram positive organisms causing surgical site infections.

Table.1 The common samples collected from SSI patients

\begin{tabular}{|l|r|}
\hline Wound swab & $67.6 \%$ \\
\hline Pus cells & $23.8 \%$ \\
\hline Tissue cells & $5.3 \%$ \\
\hline Synovial fluid & $0.7 \%$ \\
\hline Bile C/S & $0.7 \%$ \\
\hline Breast discharge & $0.7 \%$ \\
\hline Pancreatic Pseudocyst & $0.7 \%$ \\
\hline
\end{tabular}


Table.2 Etiological agents causing SSI

\begin{tabular}{|l|c|}
\hline Microorganism & \% of isolates \\
\hline E. coli & $26.1 \%$ \\
\hline Klebsiella pneumonia & $17.6 \%$ \\
\hline Staphylococcus aureus + MRSA & $12.3 \%$ \\
\hline Pseudomonas aerugenosa & $6.9 \%$ \\
\hline Actinoebacter baumanii & $5.3 \%$ \\
\hline Proteus mirabilis & $5.3 \%$ \\
\hline Enterococcus & $4.6 \%$ \\
\hline Citrobacter & $2.3 \%$ \\
\hline CONS & $1.5 \%$ \\
\hline Morganella morganii & $1.5 \%$ \\
\hline Enterobacter Cloacae & $1.5 \%$ \\
\hline Actinobacter SPP & $0.7 \%$ \\
\hline
\end{tabular}

Table.3 Antimicrobial susceptibility pattern of etiological agents causing SSI

\begin{tabular}{|l|l|}
\hline Microorganism & \multicolumn{1}{c|}{ Resistance } \\
\hline E. coli & Cu,A, Cz, Cot, Ctr, Cpm,Cip, G \\
\hline Klebsiella pneumoniae & A, Ctr, Cpm, Cot, AK, G, Cfs, Cip \\
\hline Staphylococcus aureus & Cot, Cip, G \\
\hline Pseudomonas aerugunosa & G, Cip \\
\hline Acitinoebacter baumanii & Pit, Cfs, I, Mrs, Tgc \\
\hline CONS & E, CD, Cip, G \\
\hline Enterococcus faecalis & P, Cox, Cot, Ctz \\
\hline Provedentia staurtii & G,AK, Ctr, Pit \\
\hline Proteus mirabilis & A, Ctr, Cpm, I, Cot,Cl, G \\
\hline MRSA & Cot, Cip, G, P, Of \\
\hline Morganella morganii & G, Ctr, Cot, Tgc, Cl \\
\hline Citrobacter & A, Cip, I \\
\hline Enterobacter cloacae & A, Ctr, Cpm, Cot, Cu, G, Cfs, Cip, Ct, I, \\
\hline
\end{tabular}

Table.4 Antimicrobial susceptibility pattern of etiological agents causing SSI

\begin{tabular}{|l|l|}
\hline Microorganism & Sensitivity \\
\hline E. coli & Caz, I, Mrp, AK, Cfs, Pit, Cof \\
\hline Klebsiella pneumoniae & A \\
\hline Staphylococcus aureus + MRSA & Va, Lz, A, Of, E, CD \\
\hline Pseudomonas aerugunosa & Cpm, Caz, I, Mrp, Ak, Cfs, Pit, Cof \\
\hline Acitinoebacter baumanii & Cpm, Caz, AK, Cof \\
\hline
\end{tabular}


Figure.1 The common specimens collected from SSI patients

$\begin{array}{lll}\text { Wound Swab } & \text { Pus cls } \\ \text { Synovial fluid } & \text { Bile C/S } & \text { Tissue cls } \\ \text { Pancreatic pseudocyst } & & \end{array}$

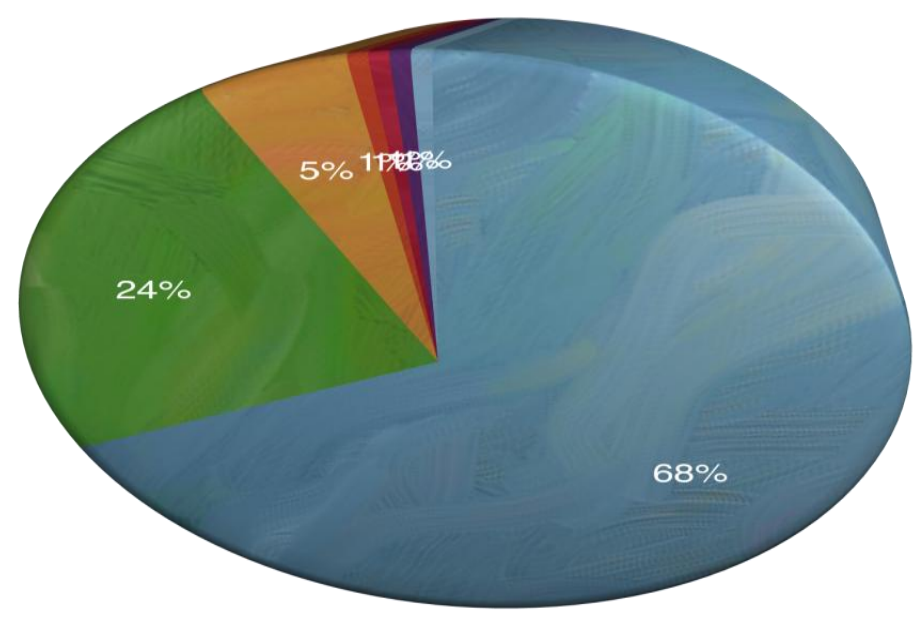

Figure.2 The common organisms collected from SSI patients

E.coli

Acitinoebacter baumanii

Citrobacter

Enterobacter cloacae
Klebsiella pneumoniae

Proteus mirabilis

CONS

Provedentia stuartii
Staphylococcus + MRSA

Enterococcus

Morganella morganii

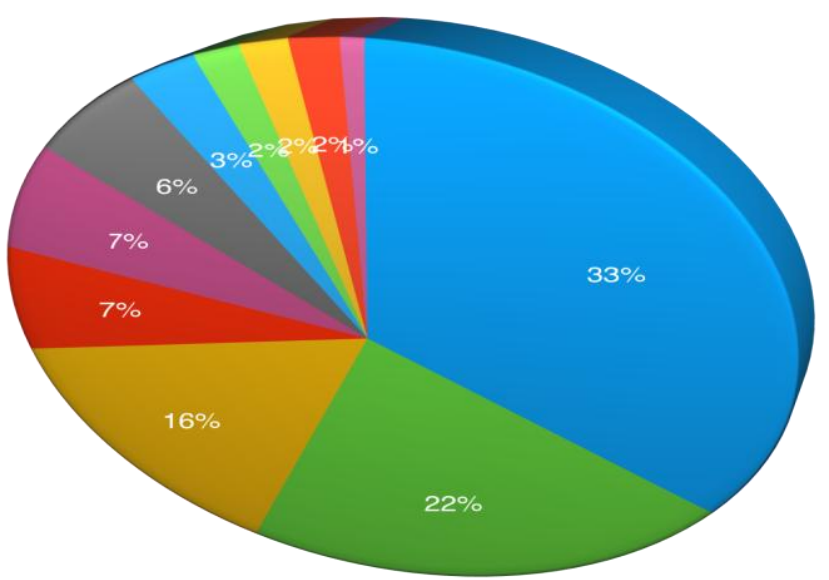

Surgical site infection is becoming common in day to day practice. There is an increased rate of SSI observed in hospital settings.

This study mainly focuses on the common organisms associated with wound infections and their susceptibility pattern so that antibiotics can be started propylactically based on antibiogram and would reduce the duration of hospital stay and minimise the cost. The study was conducted at Department of General Surgery, Department of Microbiology of Saveetha Medical College.

This is a retrospective study of 130 cases. Comparing among our case setting and of that done by Hemant Borse et al., the most common micro-organism causing SSI is $E$. coli and in our next most common isolated from SSI is Klebsiella sps...in contrary to 
their study where Pseudomonas was the next most commonest organism isolated in their hospital settings. In our study most of the gram negative microorganisms were sensitive to imipenem, amikacin in concordance with their study which concludes Imipenem and amikacin can be started prophylactically for patients before surgical procedures.

Comparing among our case setting performed on 130 patients and that of Vikrant Negi et al., performed on 768 patients the most common organism was Staphylococcus aureus which was the fourth most common organism in our case setting. Most of the organisms were sensitive to vancomycin, teicoplanin, linezoid which were similar to our case setting. Majority of the Gram positive organisms were Methicillin resistant staphylococcus aureus were mainstay of treatment would be vancomycin and linezolid.

Comparing among our case setting performed on 130 patients and that of Mundhada et al., performed on 100 patients, the most common organism was Staphylococcus aureus which was the fourth most common organism in our case setting. None of the strains were Methicillin resistant, but in our case setting some strains were Methicillin resistant.

Comparing among our case setting to that of done by Setty et $a l$., in a government tertiary care teaching hospital in Mysore, Karnataka, the most common organism causing SSI is Staphylococcus aureus which was the fourth most common organism in our case setting.

Among all the studies carried out on SSI the most commonly isolated organisms in various hospital settings were E. coli, Klebsiella sps, Pseudomonas, Staphylococcus aureus, and Acinetobacter. Both MRSA and MSSA were isolated from those patients. Multi drug resistance is increasingly noted with all those isolates. On analysing the antibiogram of those isolates carbapems would be a better drug for gram negative coverage and vancomycin and licizolid would be a better drug for gram positive coverage.

Though anaerobic organisms are common with wound infections this study mainly focuses on aerobic organisms.

In conclusion, this is a retrospective study carried out on 130 patients with SSI in Saveetha medical college and hospital, thandalam. The most common microorganism causing surgical site infection in our hospital setting was $E$. coli. The next most common organisms were Klebsiella, pseudomonas and Staphylococcus aureus.

On analysing the antibiogram and their susceptibility pattern, carbapenems are preferred drug for gram negative coverage, vancomycin and linizolid are preferred for gram positive coverage. Starting these drugs prophylactically would reduce the risk of SSI, duration of post op stay and cost.

\section{References}

Anvikar AR, Deshmukh AB, Karyakarte RP, Damle AS, Patwardhan NS, Malik AK, et al. A one year prospective study of 3280 surgical wounds. Indian J Med Microbiol. 1999; 17: 129-32.

Available from: http://www.chcanys.org/ clientuploads/downloads/

Clinical_Conf_2007/TracksMay/SIPNo rthwestCollaborativeHandbook. pdf [Last cited on 2013 May 14].

Eriksen HM, Chugulu S, Kondo S, Lingaas E. Surgical-site infections at Kilimanjaro Christian Medical Center. JHosp Infect 2003; 55: 14-20.

Hemant Borse and Rahul Shelke2 Study of Various Organisms associated with Surgical Site Infection and their Sensitivity Pattern. MVP Journal of 
Medical Sciences, Vol 2(2), 118-123, July-December 2015

Lilani SP, Jangale N, Chowdhary A, Daver GB. Surgical site infection in clean and clean-contaminated cases. Indian J Med Microbiol 2005; 23: 249-52.

Mangram AJ, Horan TC, Pearson ML, Silver LC, Jarvis WR. Guideline for prevention of surgical site infection, 1999. Hospital Infection Control Practices Advisory Committee. Infect Control Hosp Epidemiol., 1999; 20: 250-78.

Morris CD, Sepkowitz K, Fonshell C, Margetson N, Eagan J, Miransky J, et al. Prospective identification of risk factors for wound infection after lower extremity oncologic surgery. Ann Surg. Oncol. 2003; 10: 778-82.

Mundhada AS1, Tenpe S. A study of organisms causing surgical site infections and their antimicrobial susceptibility in a tertiary care government hospital. Indian Journal Pathology Microbiology. 2015 Apr-Jun; 58(2): 195-200. doi: 10.4103/03774929.155313.

Patel SM, Patel MH, Patel SD, Soni ST, Kinariwala DM, Vegad MM. Surgical site infections: Incidence and risk factors in a tertiary care hospital, Western India. Natl J Community Med 2012; 3: 193-6.
Reichman DE, Greenberg JA. Reducing Surgical Site Infections: A Review. Rev Obstet Gynecol 2009; 2: 212-21.

Setty NH, Nagaraja MS, Nagappa DH, Giriyaiah CS, Gowda NR, Laxmipathy Naik RD. A study on Surgical Site Infections (SSI) and associated factors in a government tertiary care teaching hospital in Mysore, Karnataka. Int J Med Public Health 2014; 4: 171-5.

Suljagić V, Jevtic M, Djordjevic B, Jovelic A. Surgical site infections in a tertiary health care center: Prospective cohort study. Surg Today 2010; 40: 763-71.

Urban JA. Cost analysis of surgical site infections. Surg Infect (Larchmt) 2006; 7(suppl 1): S19-22.

Vikrant Negi, Shekhar Pal, Deepak Juyal, Munesh Kumar Sharma and Neelam Sharma. Bacteriological Profile of Surgical Site Infections and Their Antibiogram: A Study From Resource Constrained Rural Setting of Uttarakhand State, India. Journal of Clinical Diagnostic Research. 2015 Oct; 9(10): DC17-DC20.

Watanabe A, Kohnoe S, Shimabukuro R, Yamanaka T, Iso $\mathrm{Y}, \mathrm{Baba} \mathrm{H}$, et al. Risk factors associated with surgical site infection in upper and lower gastrointestinal surgery. Surg Today 2008; 38: 404-12.

\section{How to cite this article:}

Aruthra, R and Neelusree, P. 2019. A Study of Organisms Causing Surgical Site Infections and their Antimicrobial Susceptibility in a Tertiary Care Hospital. Int.J.Curr.Microbiol.App.Sci. 8(09): 293-298. doi: https://doi.org/10.20546/ijcmas.2019.809.035 